\title{
VOLUME ESTIMATIONS FOR COMBINED FREE-WEIGHT AND RUBBER-BAND RESISTANCE EXERCISE
}

\author{
Todd C. Shoepe ${ }^{1}$, Gustavo Vejarano ${ }^{2}$, Nathan P. Reyes ${ }^{1}$, Nicole M. Gobreial ${ }^{3}$, \\ and Jeanette M. Ricci ${ }^{4}$ \\ ${ }^{1}$ Department of Health and Human Sciences, Loyola Marymount University, Los Angeles, USA \\ ${ }^{2}$ Department of Electrical Engineering and Computer Science, \\ Loyola Marymount University, Los Angeles, USA \\ ${ }^{3}$ Division of Biokinesiology and Physical Therapy, University of Southern California, USA \\ ${ }^{4}$ Department of Kinesiology, Michigan State University, USA
}

Original scientific paper

UDC: 796.015.6:519.2

\begin{abstract}
:
Volume, or the total work performed during resistance training is one of the vital variables of resistance exercise programming. The most common definition in use by practitioners is sets $x$ reps $x$ external weight. While appropriate for linear loading incurred through free-weight resistance exercise, this inadequately addresses the nonlinear loading incurred with rubber resistance, a relatively new loading technique. The purpose of this investigation was to derive a theoretical model to describe a method of volume calculation for rubber band plus free-weight exercise. Men $(n=51$; age $19.5 \pm 1.6$ years; body height $1.76 \pm 0.07$ meters; body weight $77.3 \pm 11.3$ kilograms) and women participants $(n=66$; age $18.9 \pm 1.1$ years; body height $1.65 \pm 0.07$ meters; body weight $62.8 \pm 9.1$ kilograms) were measured for band lengths incurred at: squat with knee extended position, squat with flexed position, and change in band length was then calculated. Significant gender differences were seen for band length change as a percentage of body height $(\mathrm{p}<.5)$ during the squat, which mandated separate volume equations (females $=33.8 \%$; males $=35.3 \%$ of body height). Equations were determined for total external volume estimation in $\mathrm{kgm}=\left[0.338\left(m+2 c_{2}+(\ln (h)-0.383) 2 c_{1}\right)\right] / g$ and $\mathrm{kgm}=\left[0.352\left(m+2 c_{2}+(\ln (h)-\right.\right.$ $\left.\left.0.382) 2 c_{1}\right)\right] / g$ for females and males, respectively, where $m$ is the total external resistance, $c_{2}$ and $c_{1}$ are constants derived from rubber-band loading parameters, $h$ is the body height of the participant, and $g$ is gravitational acceleration. This work provides practitioners and researchers with a simple theoretical method for work estimation using participant's body height to estimate displacement values during the squat exercise.
\end{abstract}

Key words: variable resistance, material properties, reliability, intra-repetition

\section{Introduction}

Exercise volume is one of numerous programming variables in the prescription of exercise and is important to monitor during research investigations involving exercise (Drinkwater, et al., 2005; Kramer, et al., 1997; McBride, et al., 2009; Peterson, Rhea, \& Alvar, 2004). Volume relates to the total amount of weight lifted and is the product of the number of repetitions performed and the resistance encountered (Ratamess, et al., 2009). Volume therefore speaks to the systematic quantification of mechanical work. In free-weight resistance training applications, this is interpreted as the total work performed during the concentric, gravity-dependent vertical displacement of each repetition. While methodologies involving motion capture and forceplate technologies can support the direct measure- ment of work performed (Escamilla, 2001; Israetel, McBride, Nuzzo, Skinner, \& Dayne, 2010), these methodologies present significant barriers in terms of cost, accessibility, and technician support and are unlikely to be practically useful for the strength and conditioning professional.

Total volume, or work performed, include internal factors such as the weight of the body as well as external factors such as the barbell and added plates. Many methods of volume estimation have been proposed and directly compared (McBride, et al., 2009), although most commonly, because of simplicity, practitioners primarily estimate volume by multiplying the reps, sets, and resistance to produce a total training session volume (Kraemer \& Ratamess, 2004; O'Bryant, Byrd, \& Stone, 1988; Ratamess, et al., 2009). However, this 
is a practice which neglects the measurement of displacement (a function of the participant's body height) (McBride, et al., 2009) and mass of the body segments (a function of the total body mass) during the calculation of volume and work. In practice, it can be argued that excluding displacement as a variable might present negligible effects on the error seen with volume calculations because displacement could be consistent for a given athlete and perhaps proportionately similar to different athletes. However, absolute, not proportional body height could produce significant differences in work performed during training with variable resistance. Body mass could be included in work and volume calculations via proportionate segmental masses involved in the movement (Dempster, 1955). As an example, for squats (SQ), this would include the head, upper-extremities, torso, and a portion of the thighs.

The increase in popularity of intra-repetition (Soria-Gila, Chirosa, Bautista, Chirosa, \& Salvador, 2015) variable resistance exercise applied via chains and rubber, elastic bands (Baker \& Newton, 2005; Heinecke, Jovick, Cooper, \& Wiechert, 2004; Simmons, 1996, 1999; Swinton, Lloyd, Agouris, \& Stewart, 2009; Warpeha, 2002) coupled with the varied, inconsistent adaptations reported with these loading methodologies (Anderson, Sforzo, \& Sigg, 2005; Ghigiarelli, et al., 2009; McCurdy, Langford, Ernest, Jenkerson, \& Doscher, 2009; Rhea, Kenn, \& Dermody, 2009; Shoepe, Ramirez, Rovetti, Kohler, \& Almstedt, 2011) mandates a more valid and careful examination of training volume during variable resistance applications. This is due to traditional free-weight exercises producing linear loading patterns, whereas variable resistance, as incurred by rubber-bands, exhibit nonlinear loading patterns (McMaster, Cronin, \& McGuigan, 2009; Mcmaster, Cronin, \& McGuigan, 2010; Shoepe, Ramirez, \& Almstedt, 2010; Thomas, Mueller, \& Busse, 2005). The variable loading pattern of rubber is the result of established material properties of rubber bands, which do not follow the early-phase, elastic region of Hooke's law that includes a linear elongation-force relationship.

The differences in these two loading schema provide unique challenges in two ways. The first is that common assumptions of load and volume calculations in practice for free-weight exercises compromise the validity of empirical comparisons between free-weight and variable resistance exercise. The second is that the programming variables in the prescription of resistance exercise were developed for isoinertial loading (Kraemer \& Ratamess, 2004) and therefore should be revisited for validation or possible revision to accommodate the distinctive loading patterns encountered with variable resistance exercises. Therefore, the purpose of this methodological report was to calculate and describe a method of external volume calculation for a rubber-band plus free weight exercise. A secondary purpose was to provide practitioners in the field with a method of accurately quantifying volume for combined free-weight and rubber-band loading for the barbell squat.

\section{Methods}

\section{Experimental approach}

First, a generalized equation was developed for external work performed during variable resistance training. Next, because body height is the primary factor in determining the displacement of resistance (Soria-Gila, et al., 2015), participants were recruited in order to establish normative values for displacement during the SQ exercise. These normative values were then used to develop equations for displacement as a function of body height for both men and women.

\section{Estimation of a single repetition work}

For squat exercises, work performed during any single resistance training repetition was described as follows:

$W_{T}=\int_{a}^{b} F_{T}(x) d x$

where $W_{T}=$ total work performed in joules when the practitioner stretches the band from $a$ to $b, \int=$ integral from $a$ to $b, F_{T}(x)=$ total force generated in $N$ when the band is stretched to $x, x=$ variable displacement in meters, $a=$ length of the band at the bottom in meters (e.g., femur parallel with the ground or horizontal during SQ), $b=$ length of the band at the top in meters (e.g. full hip and knee extension during SQ).

In a single free-weight repetition alone, where resistance is constant, calculating work was then only a matter of calculating the product of mass, gravity, and displacement with no further considerations. With variable resistance, the equation required a split into two parts representing the freeweight component and the rubber-band component, respectively. A graphic representation of the two components of this equation is shown in Figure 1 and includes a constant loading portion (Figure 1A) and the variable loading portion (Figure 1B).

$W_{T}=\int_{a}^{b} F_{n} d x+\int_{a}^{b} F_{e}(x) d x$

Here, $F_{n}=$ normal force in newtons $(\mathrm{N}), F_{e}=$ elastic force as a function of displacement $(x)$. With substitutions of mass and gravity for $F_{n}$, the equation was reduced further to:

$W_{T}=m g \int_{a}^{b} d x+\int_{a}^{b} F_{e}(x) d x$

Here, $m=$ total mass in kilograms of free-weights and bar and $g=$ gravitational acceleration constant. 


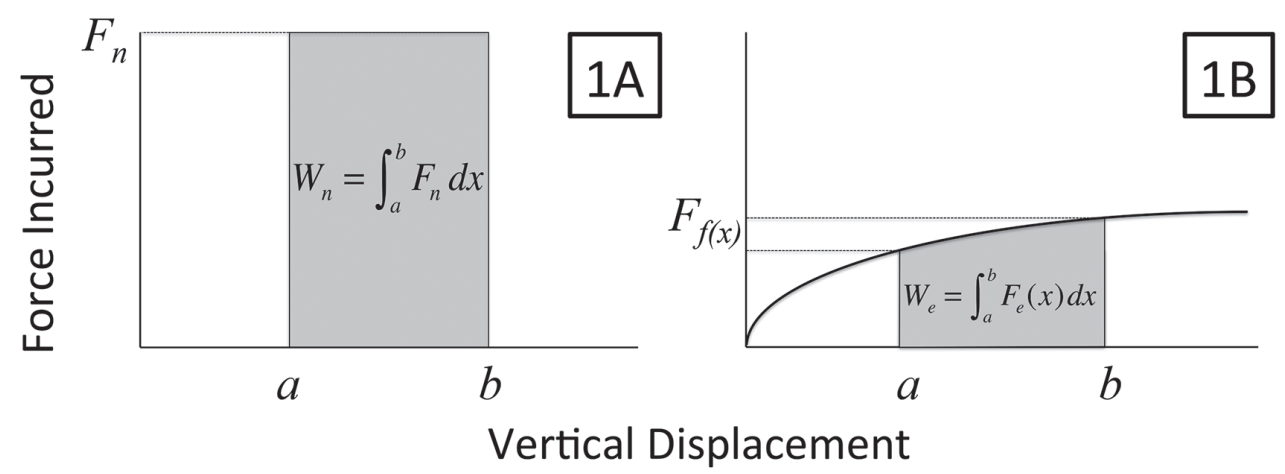

Figure 1. Graphic representation of work estimation. The work completed in overcoming resistance provided by free-weights is shown in $1 A$, while the work completed in overcoming the resistance provided by the deformation of the bands is shown in $1 B$. During a combined elastic plus free-weight condition, total work performed would be the sum of that represented in $1 A$ and $1 B$. $W_{n}=$ total normal work due to free-weight loading performed in joules, $\int=$ integral from a to $b, F_{n}=$ total force generated in $N$ due to free-weight loading, $W_{e}=$ total normal work due to elastic loading performed in joules, $\int=$ integral from a to $b, F_{f(x)}=$ total force generated in $N$ due to elastic loading, $x=$ vertical position in meters, $a=$ length of the band at the bottom in meters (e.g. parallel femur), $b=$ length of the band at the top in meters (e.g. full hip and knee extension).

Table 1. Regression equations and $R^{2}$ values for all bands for the squat attachment configurations

\begin{tabular}{lcc}
\hline Band (thickness -cm) & Regression equation* & $\mathrm{R}^{2}$ \\
\hline Orange (0.635) & $y(x)=6.44 \ln (\mathrm{x})+3.18$ & $\mathrm{R}^{2}=.962$ \\
Red (1.270) & $y(x)=10.25 \ln (\mathrm{x})+2.66$ & $\mathrm{R}^{2}=.981$ \\
Black (2.860) & $y(x)=17.08 \ln (\mathrm{x})+4.00$ & $\mathrm{R}^{2}=.983$ \\
Purple (4.450) & $y(x)=23.79 \ln (\mathrm{x})+6.60$ & $\mathrm{R}^{2}=.989$ \\
Green (6.350) & $y(x)=44.20 \ln (\mathrm{x})+11.74$ & $\mathrm{R}^{2}=.990$ \\
\hline
\end{tabular}

Note. ${ }^{*} x=$ linear band deformation in meters, $y=$ resistance applied in $\mathrm{kg}$, In = natural log, from Shoepe, et al. (2010).

Previous work (Shoepe, et al., 2010) experimentally calculated the loading properties of a set of rubberbands such that for a given length of band $(x)$, the force applied can be calculated as a function of $x$. This set of regression equations as experimentally determined is shown in Table 1.

Dividing both sides of Equation 3 by $g$, evaluating the first integral for the displacement from $a$ to $b$, and substituting the previous regression equations for band loading $y(x)$ produces:

$\frac{W_{T}}{g}=m(b-a)+n \int_{a}^{b} y(x) d x$

where $n=$ number of rubber-bands, i.e., $n=2$ for SQ.

At this time, the integral for the work contribution of rubber was solved. To do this, the logarithmic equations from Table 1 have been written in the following form, where $c_{1}$ and $c_{2}$ are constants determined by the band used by the practitioner:

$y(x)=c_{1} \ln (x)-c_{2}$

which is then used to evaluate the second integral in Equation (4) to produce:

$\int_{a}^{b} y(x) d x=c_{1}(b \ln (b)-a \ln (a))+\left(c_{2}-c_{1}\right)(b-a)$
Then, substituting Equation 6 into Equation 4 produces:

$$
\frac{W_{t}}{g}=\left(m+n\left(c_{2}-c_{1}\right)\right)(b-a)+n c_{1}(b \ln (b)-a \ln (a))
$$

At this point, work can be approximated during concurrent rubber-band and free-weight exercise using Equation 9. Given the known free-weight load including the bar, the lengths of the bands at the bottom and top of each repetition, and a type of band being used, work per repetition can be calculated using Equation 7. Direct workload comparisons were then made between the free-weightonly exercise and combined rubber band and freeweight exercise. This was accomplished using the constant part of Equation 4: $W_{T} / g=m(b-a)$, i.e., when no bands are used, $c_{1}=c_{2}=0$, and Equation 7 reduces to $W_{T} / g=m(b-a)$.

\section{Estimation of a single repetition work based on body height}

The matter of combined rubber-band and free-weight work was complicated by the need to measure the beginning $(a)$ and ending $(b)$ points of the concentric phase for each lifter. The second goal of this manuscript was therefore undertaken to eliminate the need for measuring each lifter's respective displacement for SQ and instead set out to use body height as a proportional constant in estimating the mean displacement for a given lifter with the SQ. The thought was that given someone's body height, strength and conditioning professionals could match workloads between lifters performing combined exercises and between free-weight only training sessions with one calculation and no measurements.

Participants. Prior to any involvement with human participants, approval was granted by the Institutional Review Board for the Protection of 
Human Subjects. Adult ( $>18$ years of age) participants were then recruited from the student body and written informed consent was obtained. Participants were then measured during unloaded conditions to determine band lengths at the beginning and end of the SQ.

Both the type of band and method of applying rubber-resistance to free-weight exercise can vary (Anderson, Sforzo, \& Sigg, 2008; Mcmaster, et al., 2010; Shoepe, et al., 2011). For the SQ, two bands are used on opposite sides of the bar with the free end affixed with choked knots to the squat rack support beams as displayed in Figure 2. The descriptive elements regarding loading properties of the bands apply to the five bands of varying thickness $(0.635,1.270,2.860,4.450$, and $6.350 \mathrm{~cm})$ acquired from the same manufacturer (Flex Bands, eliteFTS; London, $\mathrm{OH}$ ). For the purposes discussed later, the conclusion of the eccentric portions of the SQ are defined as $a$, while the conclusion of the concentric portion is defined as $b$. In each of these exercises, displacement $(b-a)$ was defined as the total distance moved from the end of the eccentric to the end of the concentric portions of each repetition where each of the factors is depicted in Figure 2. Calculations for band length assume that bands be placed perpendicular to the bar. However, even in the case of large placement error, the effect on band length is less than $1 \%$.

For the SQ, participants were asked to assume a standing position as displayed in Figure 2B. In the standing position, a cloth tape measure was then used to determine the length the band would be at point $b$ from the center of the bar with the participants placing the bar in the high bar position (Wretenberg, Feng, \& Arborelius, 1996) and foot positions were consistently determined in accordance with previous descriptions (Escamilla, Fleisig, Lowry, Barrentine, \& Andrews, 2001) such that stance width was approximately $130 \%$ of acromial distance. Point $b$ required an erect upright posi-

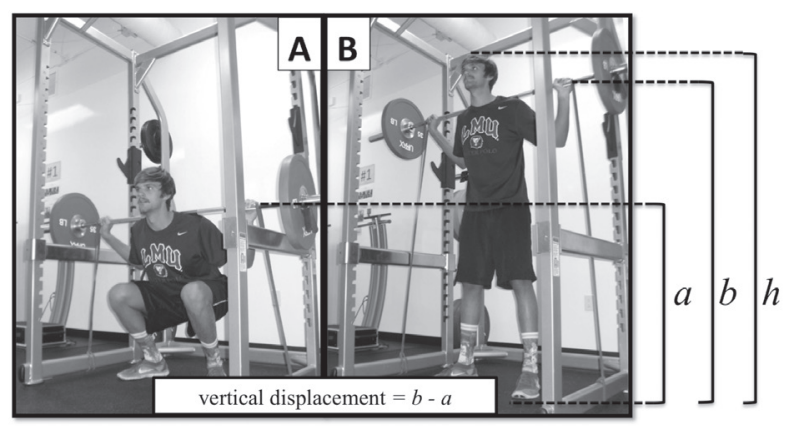

Figure 2. Band loading and bar positions for the squat (SQ). Participant position in $2 A$ identifies the conclusion of the eccentric phase, while the participant in $2 B$ depicts the conclusion of the concentric phase. Below each image is a schematic demonstrating the length of the band ( $a$ and $b$ ) as measured as it would occur in each position. For body height (h) based estimates for the substitution in equation 8 , position $a=c_{3} h$ and $b=c_{4} h$. tion with full knee and hip extension (Figure 2B), while position $a$ required hip and knee flexion with concurrent ankle dorsiflexion resulting in a parallel (horizontal) thigh position (2A) in accordance with the accepted NSCA standards of positioning and motion (National Strength Conditioning Association Certification Commission, 2008).

With equations describing the calculation of workload given a specific band thickness, and location of the lifter at the beginning and conclusion of the concentric phase of a single repetition (Equation 7), the generation of a more generic and pragmatic formula for workload would require only the body height data of the lifter. Beginning with Equation 7 sex-specific displacement data was substituted. Here, specifically $c_{3} h$ and $c_{4} h$ were replaced with $a$ and $b$ representing the location of the lifter at these critical points expressed as the mean percentage according to the body height of participants. The following two equations represent, for the first time, work done in a standard, high-bar, parallel SQ with combined free-weight and elastic resistance if the band-loading properties and approximate displacements are unknown. The result was:

$\begin{aligned} \frac{W_{t}}{g}= & \left(m+n\left(c_{\mathrm{c}}-c_{1}\right)\right)\left(c_{4} h-c_{3} h\right)+ \\ & n c_{1}\left(c_{4} h \ln \left(c_{4} h\right)-c_{3} h \ln \left(c_{3} h\right)\right)\end{aligned}$

Which was further reduced to Equation 9.

$\begin{aligned} \frac{W_{t}}{g h}= & \left(m+n\left(c_{2}+c_{1}(\ln (h)-1)\right)\right)\left(c_{4}-c_{3}\right)+ \\ & n c_{1}\left(c_{4} \ln \left(c_{4}\right)-c_{3} \ln \left(c_{3}\right)\right)\end{aligned}$

\section{Statistical analysis}

A multivariate analysis of variance was performed to examine potential between-groups differences by sex as an independent variable. Descriptive dependent variables were age, body height, and body weight, while the SQ exercise was examined for all of the following: band length at the top, band length at the bottom, band length change, band length at the top as a percentage of body height, band length at the bottom as a percentage of body height, and band length change as a percentage of body height. A value of $p<.05$ was set for statistical significance. Statistical analysis was performed with SPSS for Mac (IBM SPSS Statistics 2013, version 22.0.0.0; IBM Corp, Armonk, NY).

\section{Results}

Men $\quad(n=51 ; \quad$ age $=19.5 \pm 1.6$ years; body height $=1.76 \pm 0.07$ meters; body weight $=77.3 \pm 11.3$ kilograms) were statistically different from women $(n=66$; age $=18.9 \pm 1.1$ years; body height $=1.65 \pm 0.07$ meters; $62.8 \pm 9.1$ kilograms) in age $(p=.029)$, body height $(p<.001)$, and weight $(p<.001)$. Sex differences were seen even after body height normalizations (Table 2) for displacement and positions 
Table 2. Band positions and excursions and normalized for body height

\begin{tabular}{lcc}
\hline & Men $(\boldsymbol{n}=\mathbf{5 1})$ & Women $(\boldsymbol{n}=\mathbf{6 6})$ \\
\hline Acromial width (meters) & $0.46 \pm 0.05^{*}$ & $0.42 \pm 0.04$ \\
Band length (meters) at the top $(b)$ & $1.52 \pm 0.06^{*}$ & $1.41 \pm 0.07$ \\
Band length (meters) at the bottom $(a)$ & $0.90 \pm 0.06^{*}$ & $0.85 \pm 0.05$ \\
Band length change (meters) $(b-a)$ & $0.62 \pm 0.06^{*}$ & $0.56 \pm 0.06$ \\
Band length as a percentage of body height at the top $(b / h)$ & $86.6 \pm 2.3 \%$ & $85.8 \pm 2.6 \%$ \\
Band length as a percentage of body height at the bottom $(a / h)$ & $51.4 \pm 2.9 \%$ & $52.0 \pm 2.4 \%$ \\
Band length change as a percentage of body height $((b-a) / h)$ & $35.3 \pm 3.5 \%^{*}$ & $33.8 \pm 3.1 \%$ \\
\hline
\end{tabular}

Note. 'Statistical difference by sex $(p<.05)$.

( $p=.023$ ), both factors that influence the amount of work performed. This mandated the need to proceed with two separate equations for males and females.

Mean participant body height and variance for men and women are virtually identical to previously published U.S. standards (McDowell, Fryar, Ogden, \& Flegal, 2008) suggesting some degree of generalizability to broader populations. The quantified SQ band lengths were $86.6 \%$ for men and $85.8 \%$ for women participant's body height in position $b$, and mean values for position $a$ of $51.4 \%$ in men and $52.0 \%$ in women. The difference between these mean values for $b$ and $a$ for SQ is in direct agreement with previous work showing bar displacement during parallel squats with moderate stance widths to range from $29-38 \%$ of participant's body height (Donnelly, Berg, \& Fiske, 2006; Escamilla, et al., 2001). Continuing, these values $a=c_{3} h=0.514 h, b=c_{4} h=0.866 h$ for men and $a=c_{3} h=0.52 h, b=c_{4} h=0.858 h$ for women, and $n=2$ can be input into Equation 9 and reduced to:

$\frac{W_{T}}{g}=0.352 h\left(m+2 c_{2}+(\ln (h)-0.382) 2 c_{1}\right)$

Eq. $(10 \mathrm{~m})$

for men and:

$\frac{W_{T}}{g}=0.338 h\left(m+2 c_{2}+(\ln (h)-0.383) 2 c_{1}\right)$

for women for the SQ condition with both answers provided in units of kilogram $\bullet$ meters. The conclusion of these substitutions and equation reductions can then be applied per band revealing the equations displayed in Table $3 \mathrm{~m}$ and Table $3 \mathrm{f}$.

\section{Discussion and conclusions}

A major factor in undertaking this study was the intention of reducing the error of external workload and volume estimates that occurs during a combined rubber-band and free-weight loading exercise. Additionally, total work of the muscular system would require overcoming body or segmental mass of the body along with the external loading from free-
Table 3f. Work calculations for squats-women

\begin{tabular}{ll}
\hline $\begin{array}{l}\text { Example } \\
(\mathrm{kgm})\end{array}$ & $\frac{W_{T}}{g}=0.338 h\left(m+2 c_{2}+(\ln (h)-0.383) 2 c_{1}\right)$ \\
\hline $\begin{array}{l}\text { Orange } \\
(\mathbf{0 . 6 3 5} \mathbf{~ c m})\end{array}$ & $\frac{W_{T}}{g}=0.338 h(m+6.36+(\ln (h)-0.383) 12.88)$ \\
$\begin{array}{l}\text { Red } \\
(1.270 \mathbf{~ c m})\end{array}$ & $\frac{W_{T}}{g}=0.338 h(m+5.32+(\ln (h)-0.383) 20.5)$ \\
$\begin{array}{l}\text { Black } \\
(2.860 \mathbf{c m})\end{array}$ & $\frac{W_{T}}{g}=0.338 h(m+8.00+(\ln (h)-0.383) 34.16)$ \\
$\begin{array}{l}\text { Purple } \\
(4.450 \mathbf{c m})\end{array}$ & $\frac{W_{T}}{g}=0.338 h(m+13.2+(\ln (h)-0.383) 47.58)$ \\
$\begin{array}{l}\text { Green } \\
(6.350 \mathbf{c m})\end{array}$ & $\frac{W_{T}}{g}=0.338 h(m+23.48+(\ln (h)-0.383) 88.4)$ \\
\hline
\end{tabular}

Note. $h=$ body height of an individual in meters, $m=$ total freeweight resistance applied in $\mathrm{kg}, \ln =$ natural $\log , c_{1}$ and $c_{2}=$ constant from elastic band specific regression equations from Shoepe, et al. (2010).

Table $3 \mathrm{~m}$. Work calculations for squats-males

\begin{tabular}{ll}
\hline $\begin{array}{l}\text { Example } \\
(\mathrm{kgm})\end{array}$ & $\frac{W_{T}}{g}=0.352 h\left(m+2 c_{2}+(\ln (h)-0.382) 2 c_{1}\right)$ \\
\hline $\begin{array}{l}\text { Orange } \\
(\mathbf{0 . 6 3 5} \mathbf{~ c m})\end{array}$ & $\frac{W_{T}}{g}=0.352 h(m+6.36+(\ln (h)-0.382) 12.88)$ \\
$\begin{array}{l}\text { Red } \\
(1.270 \mathbf{~ c m})\end{array}$ & $\frac{W_{T}}{g}=0.352 h(m+5.32+(\ln (h)-0.382) 20.5)$ \\
$\begin{array}{l}\text { Black } \\
(2.860 \mathbf{c m})\end{array}$ & $\frac{W_{T}}{g}=0.352 h(m+8.00+(\ln (h)-0.382) 34.16)$ \\
$\begin{array}{l}\text { Purple } \\
(4.450 \mathbf{c m})\end{array}$ & $\frac{W_{T}}{g}=0.352 h(m+13.2+(\ln (h)-0.382) 47.58)$ \\
$\begin{array}{l}\text { Green } \\
(6.350 \mathbf{c m})\end{array}$ & $\frac{W_{T}}{g}=0.352 h(m+23.48+(\operatorname{In}(h)-0.382) 88.4)$ \\
\hline
\end{tabular}

Note. $h=$ body height of an individual in meters, $m=$ total freeweight resistance applied in $\mathrm{kg}, \ln =$ natural $\log , c_{1}$ and $c_{2}=$ constant from elastic band specific regression equations from Shoepe, et al. (2010).

weight and rubber bands. We will address external loading calculation first. 




Figure 3. Graphic representation of error in work and volume estimations. The top left panel (3A) shows hypothetical overestimation error resulting from using the force provided by the bands at the conclusion of the eccentric phase as a constant in estimating work. The bottom left panel ( $3 B$ ) shows hypothetical underestimation error resulting from using the force provided by the bands at the conclusion of the concentric phase as a constant in estimating work. The right panel (3C) shows the result of using the average force provided by the bands from a to $b$ as a constant in estimating work. $F_{a}=$ total elastic force generated in $N$ at point $a, F_{b}=$ total elastic force generated in $N$ at point $b, F_{\text {avg }}=$ total elastic force generated in $N$ at point the average force encountered from $a$ to $b, x=$ vertical position in meters, $a=$ length of the band at the bottom in meters (e.g. parallel femur), $b=$ length of the band at the top in meters (e.g. full hip and knee extension).

Naturally, error comes with estimation, and the multiple methods used to estimate workload due to the combined rubber-band and free-weight training present different magnitudes and direction of error. While free-weight load is constant and can be easily calculated, band loading is not constant, and therefore, identification of band resistance is challenging. Strength and conditioning practitioners use three common methods for estimating work completed during integrated elastic and free-weight exercise. These include using the resistance of the band treated as a constant at 1) the end of the concentric phase, 2) the end of the eccentric phase, and 3) as an average based midway between the eccentric and concentric phases. All of these methods produce systematic error in true work calculation and to aid in this discussion, a graphic examination of three methods is provided in Figure 3. When resistance due to the rubber-bands in the lock out position for SQ (e.g., extended hip and knee position) is equated to a free-weight load during volume estimation (e.g., sets x reps x resistance) as described by Shoepe et al. (2011), the result would hypothetically be an overestimation of total volume (Figure $3 \mathrm{~A}$ ) incurred during banded conditions. In this case, the rubber-band resistance is treated as a constant when the resistance decreases during the eccentric phase. Oppositely, a hypothetical underestimation occurs if the resistance provided by the rubber-band at the start of the concentric phase is used in work volume estimations (Figure 3B). Using a method of average resistance, as suggested by Anderson et al. (2008), would minimize error seen with either extreme, but due to the shape of the length-tension curves of rubber-bands, a systematic underestimate would still occur (Figure 3C). This underestimate is further exaggerated at lower band lengths when the loading curves are steeper. Given the range of band lengths that are likely to be encountered with combined loading, error could be significant according to the published band length deformation curves (Mcmaster, et al., 2010; Shoepe, et al., 2010). Overestimation would therefore be more pronounced in lifters of a smaller stature and results in sex specific differences in volume estimation where women would likely encounter a greater error than men on average. A systematic exercise-specific overestimation would also occur during exercises that deform the bands to a lesser degree such as in the SQ exercise as the lifter operates at a lower overall band length compared to a single banded bench press (BP) exercise where the band runs from one end of the bar, under the bench, and attaches to the other side of the bar. A banded configuration such as this can produce band lengths twice resting (unpublished data). This can be seen by using the reference ranges for males of $1.077 h-0.556 h$ for BP and $0.866 h-0.514 h$ for SQ. For a range of 1.5-2.0 meters in lifter's body height, a BP would produce maximum band lengths of 1.6-2.1 meters, whereas the SQ would produce the maximum band lengths of 1.3-1.7 meters.

As a matter of example, it can be ascertained that in comparing two scenarios (free-weight only versus a combined rubber-bands and free-weight loading scheme) the error could be significant. According to Wallace, Winchester, \& McGuigan (2006), combined exercise differences from freeweight only exercise in a range where the rubberband load is $20-35 \%$ of the total. Therefore, a man of 1.8 meters in body height performing a combined rubber-band plus free-weight repetition would encounter $23 \mathrm{~kg}$ of rubber-band resistance and between $43 \mathrm{~kg}$ (if rubber bands were to contribute $35 \%$ of the total) and $92 \mathrm{~kg}$ (if rubber bands were to contribute $20 \%$ of the total) of free-weight resist- 
ance at the top extended position (b), as suggested by Wallace et al. (2006). This would produce total resistance of $66 \mathrm{~kg}$ (rubber-bands contributing 35\%) and $115 \mathrm{~kg}$ (rubber-bands contributing 20\%), respectively. The resulting total real work calculated with Equation $10 \mathrm{~m}$ results in $356 \mathrm{~J}$ and $662 \mathrm{~J}$ of work, respectively. By comparison, the same lifter with an assumed constant load estimate at the top position would complete a single SQ repetition of $66 \mathrm{~kg}$ and $115 \mathrm{~kg}$ totaling $408 \mathrm{~J}$ and $714 \mathrm{~J}$ or work, respectively. As displayed in figure $3 \mathrm{~A}$, the overestimation or work performed with an assumption of constant loading with equilibrated loading in the extended position would range between $7.3 \%$ (at $35 \%$ of rubber-band loading) and $12.7 \%$ (at $20 \%$ of rubberband loading) for combined ranges, suggested by Wallace et al. (2006). As suggested in Figure 3B, using equilibrated loading at the bottom (a) position results in a systematic underestimation of work performed. The magnitude of error, however, is greatly increased due to band deformations occurring in the range of greater slopes for band stressstrain curves and ranges from $120 \%$ (39 J vs. 119 $\mathrm{J}$ ) and 205\% (68 J vs. $150 \mathrm{~J}$ ) of underestimation for $1.8 \mathrm{~m}$ tall men using black bands $(2.86 \mathrm{~cm})$.

A significant limitation of this study is the calculation of external loading factors only. While not the primary purpose of this research, the quantification of internal loads such as body mass and the contribution of work on the muscular system are also important in understanding the stresses placed on the musculoskeletal system during training. With consideration to elite lifters and near-maximal attempts, when external loading would be the dominant factor in total work performed, body weight alone can represent a proportionately large source of work for beginners, lower intensity efforts, as well as during loaded jumping exercises (Cuk, et al., 2014). While additional investigations are required to firmly describe and entrench the combined work done to overcome external and internal factors, an approximation can be made for body mass using existing models from Dempster and Gaughran $(1955 ; 1967)$. During the SQ as much as $80 \%$ of body weight may be encountered as work through the range of motion given between $a-b$ (Figure 2). This results from moving the entire torso, upper extremity, and head the entire displacement from $a-b$ in addition to a portion of the upper leg while disregarding the vertically static lower leg and foot. Because this represents a hypothetically constant load, with the $m_{b}$ representing the mass of the body with no bands attached (e.g. $c_{1}$ and $c_{2}=0$ ) then:

$\frac{W_{T}}{g}=0.352 h\left(m+0.8 m_{b}\right)$ Eq. $(11 \mathrm{~m})$

for men and the following for women:

$\frac{W_{T}}{g}=0.338 h\left(m+0.8 m_{b}\right)$
These final equations represent only approximations based on the original work of Dempster and Gaughran (1967), where the actual segmental mass values could vary significantly based on the ethnicity, age, gender, and athletic status of the lifter (De Leva, 1996). Confirming approximate internal loading based on these additional factors represents a much-needed area of future research.

\section{Practical applications}

Previous findings suggest that combined training programs can produce significant increases in strength and power in mesocycle-length timeframe as part of a periodized program with athletes (Soria-Gila, et al., 2015). In order to better support integration of the combined rubber band plus free weight training, information must be available for tracking work and therefore total training stimulus during transitions between free weight only and combined mesocyles.

The results of this study allow strength and conditioning practitioners to better prescribe variable resistance training programs using elastic bands added to free weight SQ. Specifically, this allows a direct comparison to the work completed in traditional free weight training where work can be calculated using determined displacements from Table 2 . One need only multiply total external resistance and gravity along with displacement as a percentage of body height [e.g., $(b-a) / h]$ for free-weight only loading. If total work is required, segmental masses can be added to produce:

$W_{T}=0.352 h\left(m+0.8 m_{b}\right) g$ Eq. $(12 \mathrm{~m})$

for men and:

$W_{T}=0.338 h\left(m+0.8 m_{b}\right) g$

for women. This allows practitioners greater certainty in prescribing both a) smooth transitions where workloads would be similar to the previous cycle, or b) significant alterations in workload during a programmed mesocycle.

Training volume is one of a variety of predictors to the morphological and physiological adaptations from exercise and is used as a factor in targeting differential training goals such as power, strength, hypertrophy, and endurance (Drinkwater, et al., 2005; Kramer, et al., 1997; McBride, et al., 2009; Peterson, et al., 2004). The rise in popularity of variable resistance exercise, such as produced by the combined rubber-band plus free-weight resistance, can now be more accurately estimated through a better understanding of rubber-band loading patterns and sources of error. The external work for all combined rubber-band and free-weight exercises can be estimated independent of the number or manufacturer of bands by Equation 4. Further- 
more, using bands of the same model and manufacturer, in a similar attachment protocol as described herein, can have workloads and volumes estimated by the following equations for SQ (Equation $10 \mathrm{~m}$ and 10f), respectively. The combined internal and external work (e.g. bars and plates, band resistance, and body segment masses) can be calculated using Equations $11 \mathrm{~m}$ and $11 \mathrm{f}$, respectively.

\section{References}

Anderson, C.E., Sforzo, G.E., \& Sigg, J.A. (2005). Combining elastic tension with free weight resistance training (Abstract). Medicine and Science in Sports and Exercise, 37 (5 Supplement), S186.

Anderson, C.E., Sforzo, G.A., \& Sigg, J.A. (2008). The effects of combining elastic and free weight resistance on strength and power in athletes. Journal of Strength and Conditioning Research, 22(2), 567-574.

Baker, D., \& Newton, R.U. (2005). Methods to increase the effectiveness of maximal power training for the upper body. Strength and Conditioning Journal, 27(6), 24-32.

Cuk, I., Markovic, M., Nedeljkovic, A., Ugarkovic, D., Kukolj, M., \& Jaric, S. (2014). Force-velocity relationship of leg extensors obtained from loaded and unloaded vertical jumps. European Journal of Applied Physiology, 114(8), 1703-1714.

De Leva, P. (1996). Adjustments to Zatsiorsky-Seluyanov's segment inertia parameters. Journal of Biomechanics, 29(9), 1223-1230.

Dempster, W.T. (1955). Space requirements of the seated operator: Geometrical, kinematic, and mechanical aspects of the body, with special reference to the limbs. Retreived from: deepblu.lib.umich.edu on July 25, 2017.

Dempster, W.T., \& Gaughran, G.R. (1967). Properties of body segments based on size and weight. Developmental Dynamics, $120(1), 33-54$.

Donnelly, D.V., Berg, W.P., \& Fiske, D.M. (2006). The effect of the direction of gaze on the kinematics of the squat exercise. Journal of Strength and Conditioning Research, 20(1), 145-150.

Drinkwater, E.J., Lawton, T.W., Lindsell, R.P., Pyne, D.B., Hunt, P.H., \& McKenna, M.J. (2005). Training leading to repetition failure enhances bench press strength gains in elite junior athletes. Journal of Strength and Conditioning Research, 19(2), 382-388.

Escamilla, R.F. (2001). Knee biomechanics of the dynamic squat exercise. Medicine and Science in Sports and Exercise, $33(1), 127-141$.

Escamilla, R.F., Fleisig, G.S., Lowry, T.M., Barrentine, S.W., \& Andrews, J.R. (2001). A three-dimensional biomechanical analysis of the squat during varying stance widths. Medicine and Science in Sports and Exercise, 33(6), 984-998.

Ghigiarelli, J.J., Nagle, E.F., Gross, F.L., Robertson, R.J., Irrgang, J.J., \& Myslinski, T. (2009). The effects of a 7-week heavy elastic band and weight chain program on upper-body strength and upper-body power in a sample of division 1-AA football players. Journal of Strength and Conditioning Research, 23(3), 756-764.

Heinecke, M., Jovick, B., Cooper, Z., \& Wiechert, J. (2004). Comparison of strength gains in variable resistance bench press and isotonic bench press. Journal of Strength and Conditioning Research, 18(4), 10.

Israetel, M.A., McBride, J.M., Nuzzo, J.L., Skinner, J.W., \& Dayne, A.M. (2010). Kinetic and kinematic differences between squats performed with and without elastic bands. Journal of Strength and Conditioning Research, 24(1), 190-194. doi:10.1519/JSC.0b013e31819b7995

Kraemer, W.J., \& Ratamess, N.A. (2004). Fundamentals of resistance training: Progression and exercise prescription. Medicine and Science in Sports and Exercise, 36(4), 674-688.

Kramer, J.B., Stone, M.H., O’Bryant, H.S., Conley, M.S., Johnson, R.L., Nieman, D.C., . . Hoke, T.P. (1997). Effects of single vs. multiple sets of weight training: Impact of volume, intensity, and variation. Journal of Strength and Conditioning Research, 11(3), 143-147.

McBride, J.M., McCaulley, G.O., Cormie, P., Nuzzo, J.L., Cavill, M.J., \& Triplett, N.T. (2009). Comparison of methods to quantify volume during resistance exercise. Journal of Strength and Conditioning Research, 23(1), 106-110.

McCurdy, K., Langford, G., Ernest, J., Jenkerson, D., \& Doscher, M. (2009). Comparison of chain- and plate-loaded bench press training on strength, joint pain, and muscle soreness in Division II baseball players. Journal of Strength and Conditioning Research, 23(1), 187-195. doi:10.1519/JSC.0b013e31818892b5

McDowell, M.A., Fryar, C.D., Ogden, C.L., \& Flegal, K.M. (2008). Anthropometric reference data for children and adults: United States, 2003-2006. National Health Statistics Reports, 10. Retrieved from gkh.h-cdn.co on July $25,2017$.

McMaster, D.T., Cronin, J., \& McGuigan, M. (2009). Forms of variable resistance training. Strength and Conditioning Journal, 31(1), 50-64.

Mcmaster, D.T., Cronin, J., \& McGuigan, M. R. (2010). Quantification of rubber and chain-based resistance modes. Journal of Strength and Conditioning Research, 24(8), 2056-2064.

National Strength Conditioning Association Certification Commission. (2008). Exercise technique manual for resistance training: Step by step checklists for 57 exercises ( $2^{\text {nd }}$ ed.). Champaign, IL: Human Kinetics. 
O’Bryant, H.S., Byrd, R., \& Stone, M.H. (1988). Cycle ergometer performance and maximum leg and hip strength adaptations to two different methods of weight-training. Journal of Strength and Conditioning Research, 2(2), 27-30.

Peterson, M.D., Rhea, M.R., \& Alvar, B.A. (2004). Maximizing strength development in athletes: A meta-analysis to determine the dose-response relationship. Journal of Strength and Conditioning Research, 18(2), $377-382$.

Ratamess, N., Alvar, B., Evetoch, T., Housh, T., Kibler, W., \& Kraemer, W. (2009). Progression models in resistance training for healthy adults [ACSM position stand]. Medicine and Science in Sports and Exercise, 41(3), $687-708$.

Rhea, M.R., Kenn, J.G., \& Dermody, B.M. (2009). Alterations in speed of squat movement and the use of accommodated resistance among college athletes training for power. Journal of Strength and Conditioning Research, 23(9), 2645-2650. doi:10.1519/JSC.0b013e3181b3e1b6

Shoepe, T.C., Ramirez, D.A., \& Almstedt, H.C. (2010). Elastic band prediction equations for combined free-weight and elastic band bench presses and squats. Journal of Strength and Conditioning Research, 24(1), $195-200$. doi:10.1519/JSC.0b013e318199d963

Shoepe, T.C., Ramirez, D.A., Rovetti, R.J., Kohler, D.R., \& Almstedt, H.C. (2011). The effects of 24 weeks of resistance training with simultaneous elastic and free weight loading on muscular performance of novice lifters. Journal of Human Kinetics, 29, 93-106. doi:10.2478/v10078-011-0043-8

Simmons, L.P. (1996). Chain reactions: Accommodating leverages. Powerlifting USA, 19(12), 2-3.

Simmons, L.P. (1999). Bands and chains. Powerlifting USA, 22(6), 26-27.

Soria-Gila, M.A., Chirosa, I.J., Bautista, I.J., Chirosa, L.J., \& Salvador, B. (2015). Effects of variable resistance training on maximal strength: A meta-analysis. Journal of Strength and Conditioning Research.

Swinton, P.A., Lloyd, R., Agouris, I., \& Stewart, A. (2009). Contemporary training practices in elite British powerlifters: Survey results from an international competition. Journal of Strength and Conditioning Research, 23(2), 380-384. doi:10.1519/JSC.0b013e31819424bd

Thomas, M., Mueller, T., \& Busse, M. (2005). Quantification of tension in Thera-Band $®$ and Cando ${ }^{\circledR}$ tubing at different strains and starting lengths. Journal of Sports Medicine and Physical Fitness, 45(2), 188.

Wallace, B.J., Winchester, J.B., \& McGuigan, M.R. (2006). Effects of elastic bands on force and power characteristics during the back squat exercise. Journal of Strength and Conditioning Research, 20(2), 268-272.

Warpeha, J.M. (2002). Accommodating resistance. NSCA's Performance Training Journal, 4(2), 22-23.

Wretenberg, P., Feng, Y., \& Arborelius, U.P. (1996). High- and low-bar squatting techniques during weight-training. Medicine and Science in Sports and Exercise, 28(2), 218-224.

Submitted: August 24, 2016

Accepted: April 25, 2017

Published Online First: October 12, 2017

Correspondence to:

Todd C. Shoepe, EdD, MS, CSCS, HFS

Departrment of Health and Human Sciences

Loyola Marymount University

1 LMU Drive MS 8888

LSB 181

Los Angeles, CA 90045, USA

Phone: 310-338-7825

Fax: 310-338-7882

E-mail: tshoepe@1mu.edu

\section{Acknowlegements}

The bands referred to in this study were purchased online through eliteFTS and the authors maintained no professional affiliation with this company at the time of this submission. We would graciously like to thank Recreation Services at Loyola Marymount and Dana McCaw along with every single member of the I.N.V.E.S.T. research team including: Hawley C. Almstedt, Adam Afflalo, William Alvarenga, Noel Barragan, Ashley Boyer, Bryce Brown, Jackie Canepa, Alex Cedillo, Reese Cuddy, Sam Furie, David Kohler, Erica Hanson, Phil Higgins, Crystal Holley, Emileigh Ip, Gianna Ligouri, Brooke Lejeune-Chanman, Nicole Lopes, David A. Ramirez, Robert J. Rovetti, Landon Storaasli, Megan Taele, Jen Topor, Sammy Torres, and Sean Travis. 